\title{
11 SACADDOS: A SUPPORT TOOL TO MANAGE MULTILEVEL DOCUMENTS
}

\author{
Jérôme Carrère, Frédéric Cuppens, and Claire Saurel
}

\begin{abstract}
:
This paper describes SACADDOS, a decision support tool to derive the sensitivity of a document when this document is transmitted, and to control the evolution of this sensitivity over time. For this purpose, SACADDOS manages a set of classification security policies. A classification security policy corresponds to a set of rules which are used to derive, from the content of a document, the classification of this document at a given time. SACADDOS includes an intelligent document management tool to analyze the content of a document in order to derive which classification rules apply to this document. When several contradictory rules apply, SACADDOS suggests to solve the conflict by defining an order of preference between the contradictory rules.
\end{abstract}

\subsection{INTRODUCTION}

Many organizations, especially intelligence services, have to manage huge numbers of documents, some of them which are sensitive. Generally, these organizations define security policies to derive a classification level from the content of a document. These security policies correspond to sets of rules, for example :

1. Documents in nuclear transport must be confidential.

2. Mission plans in weapons delivery for Bosnia must be secret.

3. Mission plans about hostage liberation in Bosnia must be top secret.

Moreover, sensitivity of a document may change over time. Generally, security policies manage the evolve in classification depending on the type of the document and content. These security policies define rules, for instance: 
1. A document classified at the secret level needs to be downgraded after 10 years.

2. A document classified at the confidential level needs to be downgraded after 5 years.

3. An occasional mission plan must be downgraded the day after the completion of this mission.

These are examples of downgrading rules. These rules specify a reduction of document sensitivity. In addition, there are rules to specify that the classification of a document must be upgraded, for example:

1. In the case of a conflict with a particular country, every confidential corresponding document must be upgraded at the secret level.

Choosing the actual sensitivity of a document and defining how this sensitivity is to be changed in time requires an analysis of the documents content. When the number of documents becomes significant, determining their sensitivity becomes a long and tedious task. Therfore, in many organizations which have to deal with sensitive information, there is a clear need for a tool which provides automated capabilities for classification and downgrading of documents.

However, there has been little research done in this direction. The first system proposed by McHugh [6] provides only online assistance to a human to downgrade text. Another published paper on automatic classification/downgrading of text is by Lunt and Berson [5]. This paper describes Classi, an expert system to classify and sanitize text based upon content, context or information source. As noticed by [8], the main drawbacks of this system is that document analysis capabilities in Classi are only based on keywords and associations within a sentence. This approach does not provide sufficient understanding of "natural language" ${ }^{1}$ so that we can expect to obtain good result when automating the classification or downgrading of text.

Fortunately, for several years, "intelligent" support tools for document management have been designed. They provide functions to analyze both the syntactic and semantic content of a document. Such tools for instance, can automatically determine if a document is dealing with nuclear transport. This technique is sufficiently powerful to remove most ambiguities and to dynamicly find main concepts in the text.

The purpose of this paper is to present a tool called SACADDOS. SACADDOS automatically determines the document sensitivity and controls evolution of this sensitivity over time. It is a decision support tool which suggests classification and downgrading choices to users. It also provides explanations for this choice by providing traces of derivation and enlightening the relevant portions

$\overline{{ }^{1} \text { Common written or spoken language. }}$ 
of the text. But, of course, users are always responsible for the final decision. The basic principle of SACADDOS is to combine a module for management of security policies used to classify and change the classification of documents, with a tool for document management.

The remainder of this paper is organized as follows. Section 2 presents the main objectives and functionalities of SACADDOS. Section 3 shows how knowledge, especially classification and downgrading security policies, are represented and managed in SACADDOS. Section 4 describes its logical architecture. Finally, section 5 concludes this paper by investigating several issues in this work.

Notice that it is not the purpose of this paper to investigate the problem of enforcing high security assurance when automating the classification and downgrading processes. This is an important but complex problem which represents further work that remains to be done.

\subsection{OBJECTIVES OF SACADDOS}

The work presented in this paper applies to the context of multilevel security policies. In a multilevel security policy, every piece of information is associated with a classification level and every agent is associated with a clearance level. Classification and clearance levels are taken from a set of security levels associated with a partial order relation. The confidentiality property in the multilevel security policy states that an agent can only know a given piece of information if the clearance level of this agent is higher than or equal to the classification level of the information.

In the remainder of this paper, we shall consider four security levels: NC (public), CD (confidential), SD (secret) and TSD (top secret). This set of security levels is actually associated with a total order relation: $\mathrm{NC} \prec \mathrm{CD} \prec$ SD $\prec$ TSD. However, the work presented here also applies to the case of a partial order relation.

We shall consider that information is represented by the content of full text document in "natural language". The first problem to be solved by the transmitter of a document is to decide how to classify this document. For this purpose, we assume that some security policies have been defined to derive the classification of a document from the content of this document. Currently, classifying a document is performed by manually analyzing the document content and manually applying the classification security policies to this document. However, due to the ceaseless increase in the bulk of information and because of the existence of intelligent document management tools, it is necessary and possible to design a tool to provide agents with assistance in this classification process. This is the first objective of SACADDOS.

The classification of a document does not remain unchanged over time. After varying duration, the document content becomes obsolete and the document classification is to be downgraded. Therefore, after a document has been classified, the transmitter of a document has to fulfill the following tasks: 
1. To choose a downgrading type for this document. In accordance with the security policies, there are two types of downgrading (see also [2]):

- At a time: this corresponds to a decision of downgrading the document classification at a specific time.

- By order: in this case, the document can be downgraded only if the transmitter of this document gives the order to downgrade it.

2. If the decision is to downgrade a document at a time, then the transmitter must specify the time at which the next downgrading will occur and what will be the new classification of the document at this time. This corresponds to the definition of planning to control the evolution of document classification over time.

As mentioned in the introduction, SACADDOS is a decision support system. When a user of SACADDOS has to decide how to classify or downgrade a document, SACADDOS can advise the user by suggesting classification or downgrading choices. However, a user is not obliged to follow the suggestions of SACADDOS. The user is not even obliged to ask SACADDOS for advice. In this last case, SACADDOS simply enables this user to manually classify or downgrade documents.

Let us now describe the main functions provided by SACADDOS.

Inserting a document in the document base. When new electronic sensible documents are created, they have first to be integrated in SACADDOS's document base. Related to each document, SACADDOS builds a description form with the documents main characteristics (for performance care); depending on the structure of the document, this form may or may not be filled up automaticly. In some cases there needs to be an interactive help tool for the user. SACADDOS can then perform sensitivity management operations on documents.

Classification. Following classification policies, the first step is to provide the document with a classification level. Using the querying functionality of the document management tool, SACADDOS applies classification rules in accordance with the content of the document, in order to derive this classification level. When several different classification levels can be derived from the classification rules (this situation is called a conflict), SACADDOS can help to reduce this conflict by applying some strategies that can be combined within a meta-strategy by the user. The classification level finally approved or decided by the user is stored in the description form related to the document.

Choice of downgrading type. The downgrading type of a document is defined by the security policy according to the nature or content of the document. The process of choosing downgrading type is similar to the classification one: SACADDOS selects the applicable rules and suggests a conclusion with or without applying meta-strategies. According to the security policy we consider, the 
selected downgrading type will imply different behaviors. If the downgrading type is "at a time", there is still a related downgrading planning to fill. If it is "by order", the process is completed.

Definition of downgrading planning. To start this process, it is required that the previous step of choosing document classification is completed (possibly manually), and that the downgrading type is set to "at a time" for the considered document.

The result of the classification process is a pair $\mathrm{P}_{0}=$ (initial classification date, initial classification level). Starting with this pair, the downgrading planning process aims at defining another pair $\mathrm{P}_{1}=$ (first downgrading date, first downgrading level). Then, the downgrading planning process iterates by computing a new pair $\mathrm{P}_{2}$, and so on until the public level is achieved or there is no more rule that can be applied.

The characterization of conflict is different from the classification process. The pair corresponding to ( $i$-th downgrading date, $i$-th downgrading level) has to be unique. Therefore, there is a conflict if it is possible to derive several different pairs from the set of applicable rules, that is pairs with different downgrading dates or pairs with different downgrading levels or both.

Management of downgrading. Once the planning is built, the downgrading management consists in checking if the document is properly classified in accordance with the downgrading plan and to the current date. If not, the operator is asked for downgrading the document according to its planning.

Another aspect of downgrading management is to record downgrading orders when received and to update the classification level as specified by the order.

Updates. An update corresponds to a modification of the current classification level, the downgrading type or the downgrading planning. This update is due to new applicable rules, abrogated rules, or occurrence of events (since the occurrence of an event can activate existing rules and make them applicable to some given document). In order not to have obsolete data about document protection, SACADDOS finds for each action (e.g. inserting a rule, removing a rule, adding an event, deleting an event, modifying an event, etc.) which documents are concerned by the related modification.

\subsection{KNOWLEDGE REPRESENTATION AND MANAGEMENT}

\subsubsection{A logical framework}

Here we present the language used to describe classification and downgrading security policies. Since we want to be able to both represent knowledge and to compute derived information, our language is based upon first order logic. Within this language, we will have to represent objects (e.g. documents), events (e.g. a conflict between two countries), and also operational rules (e.g. classification rules). 
For objects and events, we take our inspiration from the object-oriented paradigm. In this framework, an entity is represented by an object. Any object belongs to a "class of objects" which is an abstraction of it. The set of classes is structured in a hierarchical way, for instance, a class may inherit from another class. By doing so, we can represent different abstraction levels. A class of objects is defined by:

- its name,

- the names of the classes it inherits from,

- its attributes; attribute values are objects (so that we can define structured objects).

In our logical language, a class of objects is represented as follows:

- name: a symbol of unary predicate.

- attribute: a symbol of binary predicate. The arguments of this predicate will respectively receive the identifier of a given object, and its corresponding value for the attribute.

A given object is identified by a constant (constants are denoted by capital letters) -different constants identify different objects. We represent any inheritance link between two classes of objects $C 1$ and $C 2$ with the following rule: $\forall x, C 1(x) \rightarrow C 2(x)$.

We call entity the highest class in the hierarchy of objects. This class entity is then split into two disjoint classes: object and event. So we have the following axioms:

$$
\begin{aligned}
& \text { - } \quad \forall x, \neg(\operatorname{object}(x) \wedge \operatorname{event}(x)) \\
& \text { - } \quad \forall x, \operatorname{entity}(x) \rightarrow(\operatorname{object}(x) \vee \operatorname{event}(x)) \\
& \text { - } \quad \forall x, \operatorname{object}(x) \rightarrow \operatorname{entity}(x) \\
& \text { - } \quad \forall x, \operatorname{event}(x) \rightarrow \operatorname{entity}(x)
\end{aligned}
$$

Class event is defined with the following attributes: reference, event type, beginning date, end date. This class is introduced in our language because many classification or downgrading rules can only be applied when some event occurs, for instance, at the end of a given mission, or at the beginning of a conflict. In this case, classes mission and conflict are to be defined as subclasses of class event.

\subsubsection{Classification and downgrading policies}

The purpose of security policies we consider is to specify rules to classify and downgrade documents. Therefore, we define classes as follows. 
- Class Document has attributes: title, reference, transmitter, content (full text in "natural language"), transmission date, downgrading type, sensitivity level, classification history, downgrading planning.

A document may be structured in parts, chapters or sections. Therefore, class Document also includes the following attributes: a set of contained Parts, a set of contained Chapters, and a set of contained Sections.

Note: The content of a given document is supposed to be invariant in our system. If the content of a document changes, then a new document having this new content is created.

Security policies are modeled as a sub-class of documents ${ }^{2}$ :

- Class Policy inherits from class Document and has the following attributes: coming into effect date, set of contained Rules.

A policy may also abrogate other policies, or parts, chapters or sections coming from other policies. Therefore, class Policy also includes the following attributes: set of abrogated Policies, set of abrogated Parts, set of abrogated Chapters, set of abrogated Sections.

Finally, a policy defines a set of rules :

- Class Rule has attributes: name, transmitter, transmission date, coming into effect date, source (structural position in the policy which may contain the rule), content (original text in natural language), and logical expression (logical representation of the rule).

According to the policies we consider, rules can be sorted into three classes:

- Classification rules, for instance: "Documents which deal with nuclear transport must be confidential."

- Downgrading type rules, for instance: "A mission plan dealing with a cancelled mission must be downgraded by order."

- Downgrading rules, for instance: "A document classified at the secret level needs to be downgraded after 10 years." Such rules are used to planning the sensitivity evolution of documents which are to be downgraded at a time.

To be able to automatically derive which classification level and when it has to be assigned to a given document, we have to give logical representations to rules. Most premisses of rules are conditions either about the content of the document (especially about its themes), or about occurrence of events. In our logical language, we introduce the following predicates:

\footnotetext{
${ }^{2}$ This modeling choice makes it possible to apply the classification/downgrading process on the documents containing the security policies.
} 
- $\quad \operatorname{classif}(\operatorname{doc}, t, n)$ : the classification level of a document doc which was transmitted at time $t$ has to be $n$.

- downgrading_type(doc, $t, c$, type): the downgrading type of the document $d o c$ transmitted at time $t$ with a classification level $c$ must be type.

- downgrade $\left(\right.$ doc $\left., t, n,\left[t_{1}, n_{1}\right]\right)$ : the document doc classified at level $n$ at time $t$ must be downgraded at level $n_{1}$ at time $t_{1}$.

- deals_with(doc, theme): the document doc deals with the theme theme.

Since all classification and downgrading rules aim at giving only one conclusion, the logical representation of rules corresponds to Horn clauses. ${ }^{3}$

We need to define an adequate representation of time in order to express the notions of date (for instance, a document transmission date) and duration (for instance, "ten years", "5 days"). Our temporal representation partly takes its inspiration from [4]. We model time as a linear and continuous time, in which we distinguish some particular points which delimit time intervals. Dates and durations are denoted by triples $\langle$ day, month, year $\rangle$. For instance, $\langle 18,2,1998\rangle$ denotes the date "February 18th, 1998", and $\langle 1,3,2\rangle$ denotes a duration of two years, three months and one day.

We also define an additive function $a d d_{-} d$ between dates and durations (e.g. to compute the date corresponding to "ten years after the date of document transmission"). The algorithm of this function is specified to fit with our human intuitive way of computing such operations, especially when taking into account variable lengths of months and years. Due to the space limitation, we cannot develop this extended work here (see [3] for a more detailed presentation).

Using this language, we now give logical representations of several rules: ${ }^{4}$

- Documents which deal with nuclear transport must be confidential.

deals_with(doc, NUCLEAR_TRANSPORT)

$\rightarrow \operatorname{classif}(\mathrm{doc}, t, C D)$

- A mission report must be downgraded by order.

deals_with(doc, MISSION_REPORT)

$\rightarrow$ downgrade_type $($ doc, $t, S D, O R D E R)$

- A document classified at the secret level needs to be downgraded to the confidential level after 10 years.

$a d d_{-} d(t,\langle 10,0,0\rangle, t 1) \rightarrow$ downgrade $($ doc $, t, S D,[C D, t 1])$

- An occasional mission plan must be downgraded the day after the end of the corresponding mission:

\footnotetext{
${ }^{3} \mathrm{~A}$ Horn clause is a clause in which there is at most one positive literal.

${ }^{4}$ The constants $S D, C D, N C$ respectively represent the secret, confidential and public classification levels.
} 


$$
\begin{gathered}
\text { mission_plan }(\text { doc }) \wedge \text { mission }(m) \wedge \text { end }(m, t) \wedge \\
\text { add_d }\left(t,\langle 1,0,0\rangle, t^{\prime}\right) \wedge \text { deals_with }(\text { doc }, m) \\
\rightarrow \text { downgrade }\left(\text { doc }, t, l,\left[t^{\prime}, N C\right]\right)
\end{gathered}
$$

\subsubsection{Rules applicability}

A rule can be transmitted alone, for example, by the security administrator of an intelligence service. It can also belong to a classification or downgrading policy, which has a transmitter, a transmission date and a coming into effect date (from which rules inherit). In both cases, a rule has a validity period: it can be applied only from its coming into effect date, to the date at which another policy or rule coming into effect will abrogate it.

Notice that a rule might concern documents which were transmitted before the rule coming into effect date. This means that a rule may come into effect retroactively, applying to documents which were transmitted before the coming into effect date of the rule (see the next subsection for an example).

Notice also that a rule may be abrogated. This does not mean that the rule is physically deleted. Actually, the rule is kept by SACADDOS, and SACADDOS implements a procedure to determine which rules apply at a given time, that is rules whose validity periods include this given time. When SACADDOS tries to perform a given derivation, it only considers this subset of applicable rules. This makes it possible to reason at different times, for instance past and future times.

In some cases, there is no applicable rule to classify or downgrade a given document. In such situation, SACADDOS provides the user with a "Don't know" answer. It may also happen that an applicable rule includes reference to a not yet occurred event, for instance reference to the end of a mission. If, when the rule is applied, the end of the mission is unknown, SACADDOS provides the user with an "unknown" date (actually an existential null value).

\subsubsection{Downgrade planning and classification history}

To control the sensitivity evolution of documents over time, for which the downgrading type of a document is at a time, it is necessary to compute and then to store a downgrade plan. This plan matrix forecasts when and at which classification level a document has to be downgraded.

Nevertheless, since we design a decision support tool and not an automatic tool, a downgrading operation for a document will not necessarily be performed at the scheduled time, but sometimes later (e.g., scheduled time corresponds to vacation for the security administrator). So the downgrading dates for a document may actually not fit with the content of the downgrade plan. To be able later to know or reason about the history of the document sensitivity evolution another piece of information is necessary. This additional information is a classification history, which is another matrix similar to the downgrade plan, but with a content corresponding to the real evolution of classification. Note that ideally, downgrade planning and classification history should be identical. 


\subsubsection{Update plan}

At any given time, new applicable rules may lead to update an already computed downgrade plan:

- either a new rule comes into effect (e.g., a new classification policy) dealing with documents which were transmitted before the coming into effect date of the rule (see example 1 below),

- or a rule dealing with a given event which could not be applied to documents, because of the unoccurrence of the related event, can now be applied because of the actual occurrence of the event (see examples 2 and 3 below).

In both cases downgrade planning has to be updated since the new rules have to be taken into account. Here we give some examples of such updates.

1. Let us assume that the following rule R1 comes into effect on February 1998: every document transmitted before 1990 and dealing with Bosnia must be classified at the public level. Let us consider a document D dealing with Bosnia, transmitted at the secret level in 1989. Then, due to the general downgrading rules expressed in the introduction, this document first ought to be downgraded at the confidential level on 1999, then at the public level on 2004 (see figure 11.1). When R1 comes into effect, the classification level and the downgrading planning for $\mathrm{D}$ must be updated, although D was transmitted before February 1998. However, since we cannot change the actual past, this cannot affect the sensitivity levels the document actually got before February 1998. That is to say that document D may be, at best, downgraded in February 1998.

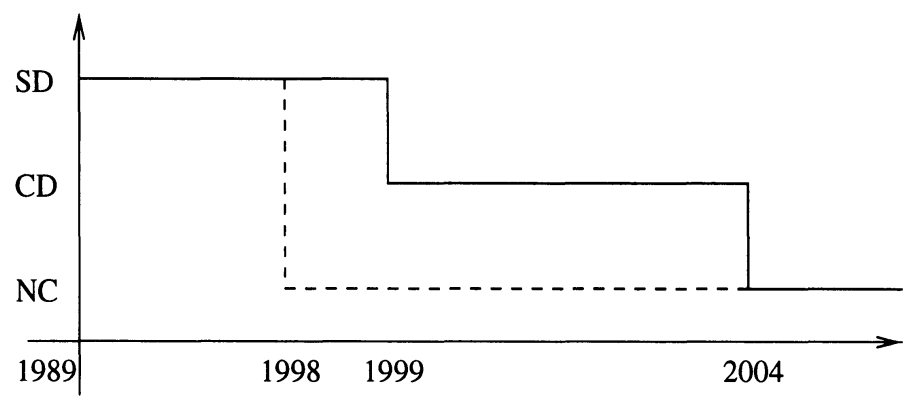

Figure 11.1 Initial planning (solid line) and updated planning (dashed line)

2. Let us assume we have the two following rules R2 and R3. R2: A mission plan has to be downgraded at a time. R3: A mission plan which deals with a mission which has been cancelled has to be downgraded by order. For a mission plan which deals with a mission which turns out to be cancelled, 
R3 must apply and will cause both an update (i.e., an adjournment) of the downgrading planning initially filled up with R2, and an update of the downgrading type.

3. Let us assume the rule: In case of conflict with a given country $X$, every confidential document about country $X$ must be upgraded at the secret level. Let us consider an American document $\mathrm{D}$, dealing with weapon delivery in Iraq, that was transmitted at the confidential level on January 1998; according to its initial downgrade plan and the rules presented in the introduction, it needs to be downgraded at the public level on January 2003. But, on February 20th, 1998, there is a conflict between USA and Iraq. So the planning must be updated; the classification of $\mathrm{D}$ must be upgraded to the secret level and the planning becomes: downgrade to the confidential level on February 20th, 2008, and then to the public level on February 20th, 2013 (see figure 11.2).

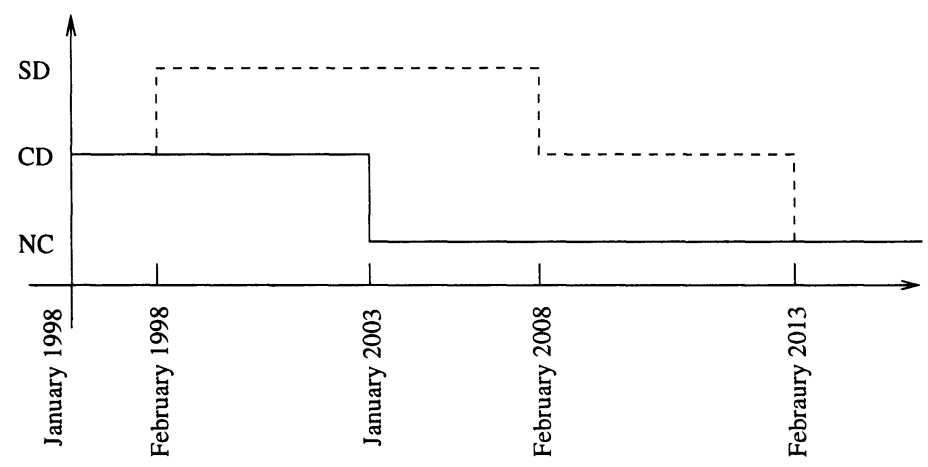

Figure 11.2 Initial planning (solid line) and updated planning (dashed line)

\subsubsection{Conflict resolution}

Suppose that at a given time, for a given document, several different rules apply giving different conclusions. Since it is assumed that at any time a given document can have only one sensitivity level, only one downgrading type and if the downgrading type is on a time, only one downgrading planning, we call such a situation a conflict. Due to the semantic nature of data classification, this problem was already identified in [7] and [5]. We distinguish three kinds of conflicts: classification level, downgrading type, and conflicts about downgrade planning.

Depending on its nature, there are several ways of managing a given conflict. It consists in defining partial preference orders between rules:

- Conflict between specific rules, coming from a same policy. Suppose a policy which regulates mission plans, and in particular mission plans about 
some sensitive countries, with different particular objectives (hostage liberation, weapon delivery...). A first strategy consists in preferring the conclusions derived from the rules whose premisses express the most specific conditions about document content.

- Conflict between rules coming from different specific policies. Suppose you have a general policy (including rules which specify downgrading after 5 or 10 years), and a policy concerning documents dealing with occasional missions. In this case, the second policy is more specific than the previous one, yet another strategy consists in preferring the conclusion coming from the most specific policy.

- Conflict between derived sensitivity levels: in some applications, a good strategy is to prefer to minimize security risk by choosing a document assignment level at the highest level.

- Conflict between downgrading dates for the same sensitivity level: another strategy consists in preferring to minimize security risk by choosing the latest date of downgrading.

- Conflict between "old" rules: one may prefer to choose the rule which came into effect most recently.

- Conflict between rules which were transmitted by an author with more or less high rank: one may prefer to choose the rule coming from the author with the highest rank.

- $\quad$ and so on...

As one can see, the preference order between rules can be defined upon the conclusions they give, and upon some intrinsic characteristics of the rules, which are stored in the rule descriptions (see section 11.3.2). So we have provided some corresponding basic strategies as the ones described below, which could be enriched by users. Since a strategy fits with a partial order defined on rules, its application aims at reducing the number of proposed conclusions for a given document, by preferring a subset of the set of applicable rules. In combining several strategies according to the choice of the user ${ }^{5}$, we obtain a meta-strategy. Using meta-strategies, it becomes possible to gradually reduce the set of suggested conclusions and sometimes get only one solution. This conflict resolution provides a help to users who in some cases can suggest to add some rules specific to their application but not included in the rule base of SACADDOS.

Due to space limitation, we do not further develop the problem of conflict resolution in this paper, but see [1] for a more detailed presentation and formalization of this problem.

\footnotetext{
${ }^{5}$ Since SACADDOS is a decision support tool, users are free to choose to apply or not basic strategies in whichever order.
} 
In summary, for a given document and a given functional operation of SACADDOS to be performed on this document, our process includes three consecutive steps:

1. selection of all rules for which the validity period includes the time of the evaluation,

2. from these rules, a selection of applicable rules for the given document (i.e., making all rule premisses true),

3. if these applicable rules give only one common conclusion, this defines the suggested result, if not, then strategies apply to reduce the set of suggested conclusions.

\subsection{LOGICAL ARCHITECTURE OF SACADDOS}

SACADDOS was designed as a client/server application. The whole core of SACADDOS runs on the server. On the client side runs only the Graphic user interface. The core of SACADDOS is composed of a knowledge base, a document database, a database for events management, and user profile descriptions. The graphic user interface runs independently from the server on a separate computer. This architecture requires us to define communication mechanism between the different modules, especially between the core of SACADDOS and the user interface.

\subsubsection{SACADDOS module description}

We first present the SACADDOS core and then the user interface. Each knowledge item considered in SACADDOS is represented as an object. The implementation is in PROLOG and keeps this principle of object knowledge representations.

11.4.1.1 Knowledge base management. The SACADDOS knowledge base includes: security policies, strategies and meta-strategies for conflict solving process. The SACADDOS knowledge base management includes processes for classification and downgrading management process for update management. The overall knowledge is managed with PROLOG.

11.4.1.2 Database for event management. Events are considered in SACADDOS as special objects that can influence the applicability of classification or downgrading rules. Ideally, the set of events may be managed by an object-oriented database management system. However, in the present prototype, they are simply simulated by a set of PROLOG facts.

11.4.1.3 Document database. The document database is managed by the document management tool. SACADDOS queries this tool to create new 


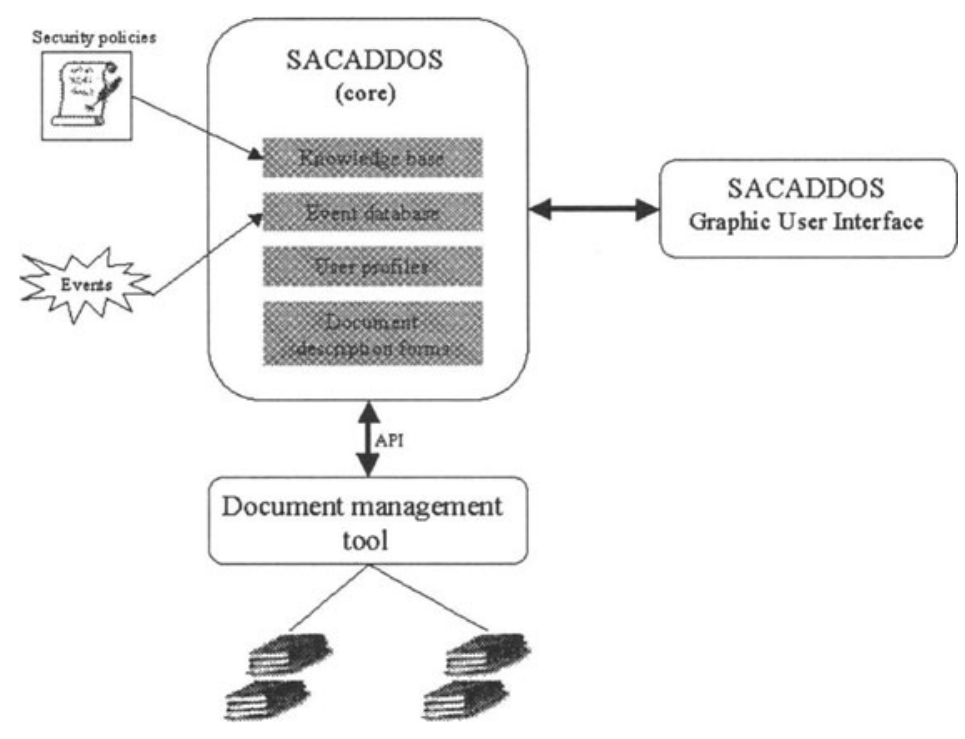

Figure 11.3 SACADDOS logical architecture

document description forms ${ }^{6}$ which include all information required to apply classification and downgrading rules. For this purpose, the document management tool selects all the themes mentioned in the document and which are involved in the rules and sends them to SACADDOS. If they are provided in the document, SACADDOS can automatically fulfill other required attributes in the document description form such as the document type, reference, transmission date and transmitter. If the document management tool does not find some information in the document file, SACADDOS warns the user about this so that the user can manually insert the missing information.

11.4.1.4 User profile management. User profiles are introduced to manage different users that are playing different roles in SACADDOS with multiple clearances. User profiles are used to restrict access to classified documents or to any other classified item stored in SACADDOS. For instance, a user with clearance secret cannot have an access to top secret documents. We also assume that the document description form has the same classification as the document it comes from. SACADDOS performs access controls so that the user only receives information it is cleared to observe according to its user profile.

${ }^{6}$ These forms are created for efficiency purpose. 
11.4.1.5 Graphic user interface. We designed an OSF/Motif based graphic user interface that locks/unlocks functionalities according to the user profile. The user interface adapts to the users who have authorization to work in SACADDOS.

\subsubsection{Communications between different modules}

The communication protocol depends on the modules to be connected:

1. Between the graphic user interface and $S A C A D D O S$ core, the message sent is directly a PROLOG query. SACADDOS core then sends preformatted results to the user-interface. Communication between that Graphic user interface and the core of SACADDOS is based on sockets.

2. Between $S A C A D D O S$ core and the document management tool, the communication is through API provided by the document management tool. Depending on the content of classification and downgrading rules, SACADDOS generates queries to be evaluated by the document management tool.

\subsection{CONCLUSION}

All the functionalities of SACADDOS which have been described in this paper are implemented. We have tested and compared several document management tools before choosing the one that will be used in SACADDOS. We started with a tool that only provided keyword oriented analysis and the possibility to define networks of concepts. However, this comes out to be not sufficient for SACADDOS. Therefore, it is currently being changed by a new system which includes a module for semantic analysis of full text documents. Using this system should greatly enhance the capability of SACADDOS. Due to modularity and since most work is performed on document description forms instead of documents themselves, there is no difficulty in changing the document management system used in SACADDOS. SACADDOS is being tested by security officers; the downgrading function ought to incite them to actually downgrade over-classified documents, that was not the case up to now.

There are several issues to this work. A first issue is that SACADDOS does not take into account subjective rules, i.e. rules whose conditions require evaluation of a user's subjective judgment. Another issue of this work would be the problem of sanitizing a document, that is retrieving a lower classified subpart from a highly classified document.

We are investigating several evolutions of SACADDOS. A first one is a module called COLCHIC, which is currently being designed. COLCHIC is based on the same principles as SACADDOS, because COLCHIC manages a set of classification and downgrading security policies and includes a document management tool. The two tools provide different functionalities, whereas, SACADDOS provides assistance to users in their task of classifying and downgrading documents, COLCHIC is a module which analyzes secondary storage memory 
to perform audit of information which are abnormally classified. COLCHIC can typically be used by security administrators to know what information is under or over classified in the system for which they are responsible. Another possible evolution of SACADDOS and COLCHIC would be to design a module which is inserted in a network to analyze communications and perform an audit of sensitive communications. The main problems when designing such a tool are exhaustivity and performances: this module must analyze and control every communication without hindering network performances. Finding solutions to these problems represents further work that remains to be done.

\section{Acknowledgements}

The work presented in this paper was supported by the CASSI under the Convention $95 / 7313929$.

\section{References}

[1] F. Cuppens, L. Cholvy, C. Saurel, and J. Carrere. Merging Security Policies: analysis of a practical example. In Proc. of the computer security foundations workshop, Rockport, Massassuchets, 1998.

[2] F. Cuppens and A. Gabillon. Modelling a multilevel database with temporal downgrading functionalities. In Ninth Annual IFIP WG 11.3 Working Conference on Database Security, Rensselaerville, USA, 1995.

[3] F. Cuppens and C. Saurel. Outil de classification et de déclassification de documents multiniveaux. Rapport 3/3564.00/DERI, Centre d'études et de recherches de Toulouse, 1997.

[4] Marie-Christine Fauvet, Jean-François Canavaggio, and Pierre-Claude Scholl. Expressions de requêtes temporelles dans un SGBD à objets. In Patrick Bosc, editor, 12èmes Journées Bases de Données Avancées, Cassi (France), 1996.

[5] T. F. Lunt and T. A. Berson. An Expert System to Classify and Sanitize Text. In Proceedings of the Third Aerospace Computer Security Conference, 1987.

[6] J. McHugh. An EMACS-based downgrader for SAT. In Proceedings of the 8th National Computer Security Conference, 1985.

[7] M. Shaefer. Dynamic Classification and Automatic Sanitization. In T. F. Lunt, editor, Research Direction in Database Security. Springer-Verlag, 1992.

[8] G. W. Smith. Classifying and Downgrading: Is a Human Needed in the Loop? In T. F. Lunt, editor, Research Direction in Database Security. Springer-Verlag, 1992. 